\title{
Enhancement of ferro-para transition in ethanol doped ferroelectric liquid crystals
}

\author{
A. Malik, A. Choudhary, J. Prakash, I. Coondoo, and A. M. Biradar ${ }^{\text {a) }}$ \\ Polymeric and Soft Material Section, National Physical Laboratory, Dr. K. S. Krishnan Road, New Delhi \\ 110012, India
}

(Received 30 September 2008; accepted 18 December 2008; published online 10 February 2009)

\begin{abstract}
The influence of ethanol $\left(\mathrm{C}_{2} \mathrm{H}_{5} \mathrm{OH}\right)$ doping in the electroclinic liquid crystal has been investigated using dielectric spectroscopy. A giant shift in ferroelectric $\left(\mathrm{SmC}^{*}\right)$ to paraelectric $(\mathrm{SmA})$ phase transition has been observed. After analyzing collective relaxation processes, it is confirmed that Curie-Weiss law is obeyed in the vicinity of $\mathrm{SmC}^{*}$ to $\mathrm{SmA}$ phase transition after doping with ethanol. It has been predicted that the dipole-dipole interaction due to ethanol molecules creates the uniformity in the randomized liquid crystal molecules in paraelectric phase, resulting in the increase in the ferroelectric phase in de Vries electroclinic liquid crystal materials. (C) 2009 American Institute of Physics. [DOI: 10.1063/1.3075611]
\end{abstract}

\section{INTRODUCTION}

Noticeable investigations have been carried out extensively in order to understand the complex phase transition from ferroelectric $\left(\mathrm{SmC}^{*}\right)$ to paraelectric $(\mathrm{SmA})$ phase of ferroelectric liquid crystals (FLCs) in which a chiral SmA phase exhibits a field dependent induced tilt angle and socalled electroclinic effect (ECE) which was described on the basis of symmetry arguments by Garoff and Meyer ${ }^{1,2}$ similar to ferroelectric concept by Meyer et al. ${ }^{3}$ The application of enough external electric field, parallel to the smectic layer, clamps the free rotation around long molecular axis of liquid crystal molecule and coupled with the permanent dipole moment, if present. As a consequence, the long molecular axis of molecule (the director) is tilted away from layer normal. ${ }^{2}$ This coupling of electric field and director tilt is called ECE and is characterized by electroclinic coefficient $e_{c}=C / \alpha(T$ $-T_{c}$ ), where $e_{c}$ stands for electroclinic coefficient, $C$ is the electroclinic coupling, and $\alpha\left(T-T_{c}\right)$ is the first coefficient of Landau free energy expression. ECE remained the topic of academic interest for about a decade. Thereafter, Andersson et $a l .{ }^{4}$ put forward the possible application of this effect. The potential applications of this are mainly in electro-optical devices where continuous change in tilt angle leads to the grayscale levels, ${ }^{5,6}$ optical image processing, real time holography, and optical correlators and interconnectors.

It is well established that the layer spacing decreases from $\mathrm{SmA}$ to $\mathrm{SmC}^{*}$ phase. But some materials are reported to have very little or almost no layer shrinkage in nonchiral SmA to SmC phase transition and are explained by a model proposed by de Vries, according to which the molecules are randomly distributed around a cone within one layer. ${ }^{7-9}$ Thereafter, it is reported in $\mathrm{SmC}^{*}$ to $\mathrm{SmA}$ phase transition also. ${ }^{10-15}$ The dielectric properties of such de Vries electroclinic materials have been thoroughly studied indicating that the relaxation frequency in $\mathrm{SmC}^{*}$ phase does not obey Curie-Weiss law in $\mathrm{SmC}^{*}$ to $\mathrm{SmA}$ phase transition which is

\footnotetext{
a) Author to whom correspondence should be addressed. Electronic mail: abiradar@mail.nplindia.ernet.in.
}

due to the randomization of molecular director around a cone, ${ }^{15,16}$ having $D_{\infty}$ overall symmetry and $C_{2}$ individual molecular symmetry. ${ }^{17,18}$ Few studies have also shown that the Curie-Weiss regime is far broader in FLC material exhibiting de Vries phases. ${ }^{19}$ Imperfection in alignment of dipole moments i.e., alignment of electroclinic liquid crystal (ELC) molecules, in SmA phase leaves the space for further alignment by any means which could lead to the increment in ferroelectric phase.

Here we report the dielectric studies of the alcohol (ethanol, $\mathrm{C}_{2} \mathrm{H}_{5} \mathrm{OH}$ ) doped de Vries ELC material, BDH 764E. It has been observed that by the addition of ethanol, transition temperature of $\mathrm{SmC}^{*}$ to $\mathrm{SmA}$ shifted drastically to higher temperature and in the scenario of shifted transition temperature, it obeys the Curie-Weiss law too.

\section{EXPERIMENTAL}

The commercially available liquid crystal used in our study is BDH 764E, which has the following phase sequence:

$$
\stackrel{?}{\text { Cryst }} \stackrel{28{ }^{\circ} \mathrm{C}}{\leftrightarrow} \mathrm{SmC}^{*} \leftrightarrow \mathrm{SmA} \leftrightarrow{ }^{\circ} \mathrm{C} \quad 89-92^{\circ} \mathrm{C}
$$

Cells for the present study were fabricated by using low resistant $(10 \Omega / \square)$ indium tin oxide coated $\lambda / 2$ glass substrates. Both the plates were assembled using Mylar spacer to achieve the thickness of $3.5 \mu \mathrm{m}$ and sealed with adhesion promoter after rubbing unidirectionally both the nylon $6 / 6$ polymer coated glass substrates to get homogeneous alignment. Most of the time perfect alignment was obtained by cooling liquid crystal from isotropic to room temperature very slowly $\left(1^{\circ} \mathrm{C} / \mathrm{min}\right)$ in the presence of electric field. The calibration of cells was carried out by using air and toluene as standard references. The ethanol was mixed in liquid crystal at $75^{\circ} \mathrm{C}$ below its boiling point $\left(78.5^{\circ} \mathrm{C}\right)$. The cells were filled in $\mathrm{N}$ phase by means of capillary action and sealed after filling.

The dielectric studies were carried out in an electrically shielded parallel plate capacitor by using Solartron 1260 


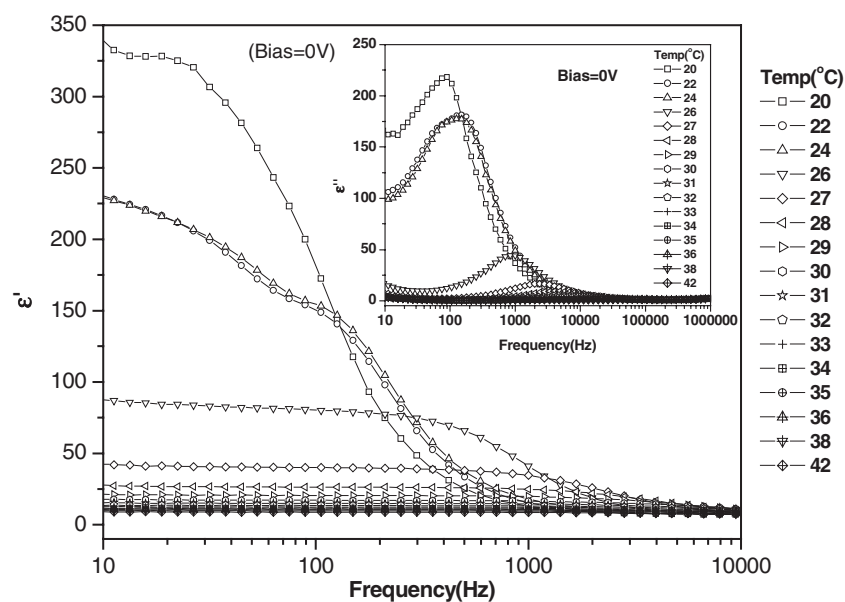

FIG. 1. Dispersion curve of dielectric permittivity ( $\varepsilon^{\prime}$ vs frequency) and in inset, dielectric absorption curves ( $\varepsilon^{\prime \prime}$ vs frequency) at different temperatures in pure $\mathrm{BDH} 764 \mathrm{E}$.

impedance/gain-phase analyzer in the frequency range of 10 $\mathrm{Hz}-10 \mathrm{MHz}$. The dielectric setup is fully computer controlled and automated. Julabo F-25 HE equipment was used for controlling the temperature with a temperature stability of $\pm 0.01{ }^{\circ} \mathrm{C}$. The sample holder was kept thermally isolated from external sources.

In the present studies, homogeneously well-aligned ELC cell has been used to perform the dielectric measurement. However, the dielectric studies have already been reported on this material, $\mathrm{BDH} 764 \mathrm{E},{ }^{15,16}$ which clearly demonstrate the nonconventional behavior of relaxation process (soft mode) and nonvalidity of Curie-Weiss law in the regime of $\mathrm{SmC}^{*}$ to SmA phase transition, which could be due to randomized paraelectric phase.

\section{RESULTS AND DISCUSSIONS}

The dielectric studies can be described in terms of the complex dielectric permittivity as given by

$$
\varepsilon^{*}(\omega, \tau)=\varepsilon^{\prime}(\omega, \tau)-j \varepsilon^{\prime \prime}(\omega, \tau),
$$

where $\varepsilon^{\prime}$ denotes the real part of the complex dielectric permittivity, $\varepsilon^{\prime \prime}$ is the imaginary part, and $\omega$ is the angular frequency of applied electric field. In order to characterize the temperature dependence of the observed dielectric relaxations, $\varepsilon^{\prime}(\omega, \tau)$ and $\varepsilon^{\prime \prime}(\omega, \tau)$ can be described by the Debye formula as follows:

$$
\varepsilon^{\prime}(\omega, \tau)=\varepsilon_{\infty}+\frac{\left(\varepsilon_{0}-\varepsilon_{\infty}\right)}{\left(1+\omega^{2} \tau^{2}\right)}
$$

and

$$
\varepsilon^{\prime \prime}(\omega, \tau)=\frac{\left(\varepsilon_{0}-\varepsilon_{\infty}\right) \omega \tau}{\left(1+\omega^{2} \tau^{2}\right)},
$$

where $\varepsilon_{0}$ is the static dielectric permittivity, $\varepsilon_{\infty}$ is the high frequency limit of dielectric permittivity, and $\tau$ is the relaxation time. The generalization of Debye formulation describes a single dielectric relaxation process. If the dielectric relaxation is exhibiting more than one process, then the re-
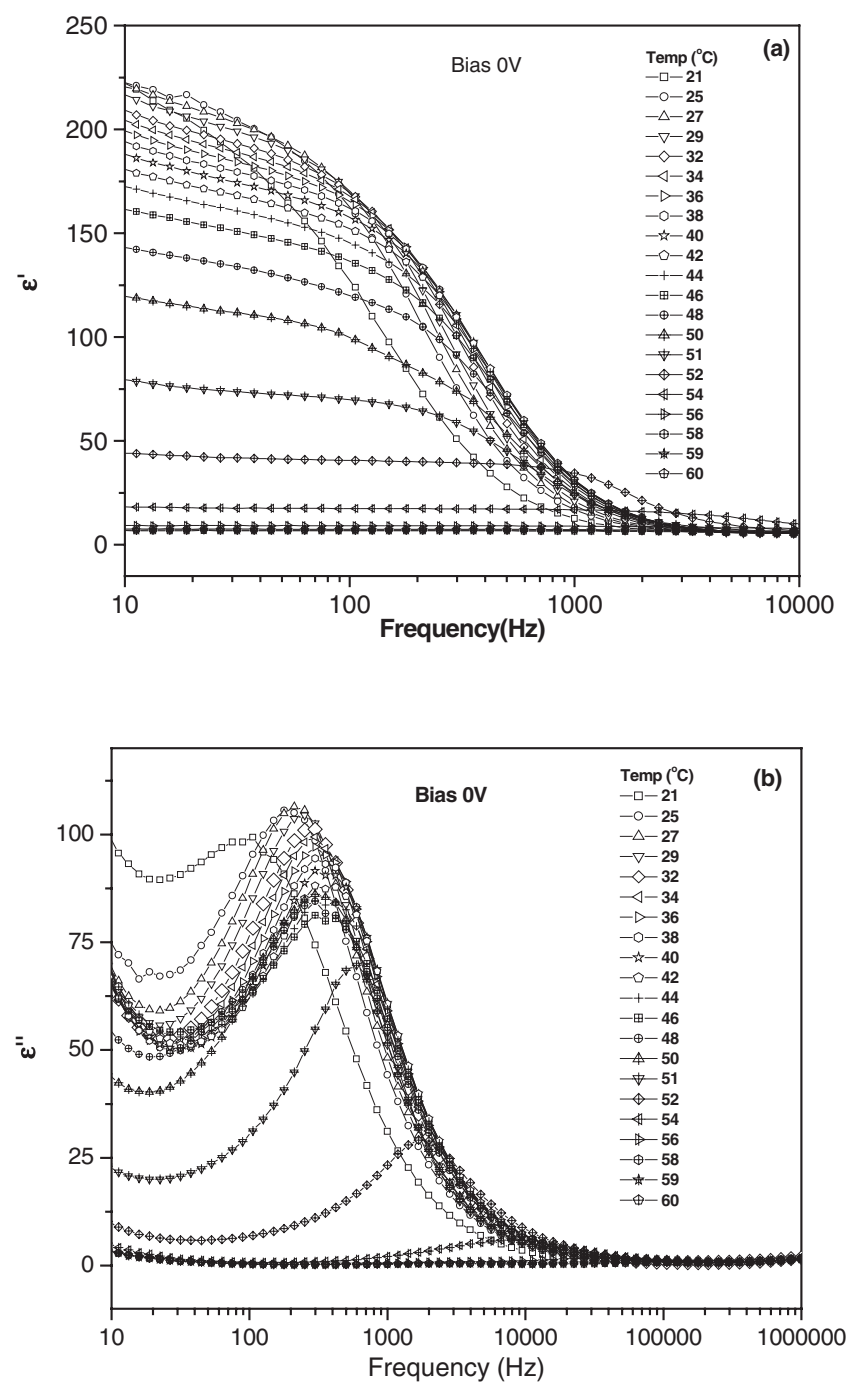

FIG. 2. (a) Dispersion curve of dielectric permittivity ( $\varepsilon^{\prime}$ vs frequency) and (b) dielectric absorption curve ( $\varepsilon^{\prime \prime}$ vs frequency) at different temperatures in ethanol doped BDH 764E.

laxation process can be described by the Cole-Cole equation as

$$
\varepsilon^{*}(\omega, \tau)=\varepsilon_{\infty}+\frac{\left(\varepsilon_{0}-\varepsilon_{\infty}\right)}{1+(j \omega \tau)^{1-\alpha}}+\frac{\sigma}{j \in_{0} \omega},
$$

where $\alpha$ is the distribution parameter for a particular relaxation process, $\in_{0}$ is the electric permittivity of free space, and $\sigma$ is the electric conductivity.

In the present investigations, two types of cells were prepared, one with ethanol mixed in ELC material and another of pure ELC, for comparative study. Figure 1 shows the dielectric permittivity as a function of frequency at different temperatures in pure ELC material. As seen in the figure, the permittivity $\left(\varepsilon^{\prime}\right)$ continuously decreases in $\mathrm{SmC}^{*}$ phase near the transition temperature $\left(28{ }^{\circ} \mathrm{C}\right)$ of $\mathrm{SmC}^{*}$ to $\mathrm{SmA}$ phase. Dielectric absorption is shown in inset of Fig. 1. The detailed dielectric process in this material is published elsewhere. ${ }^{20}$ If an ELC material is doped with some organic solvent such as $2 \%-5 \%$ of ethanol, then there is a drastic change in the dielectric relaxation process as shown in Fig. 2. The high dielectric permittivity $\left(\varepsilon^{\prime}\right)$ which is due to phason mode (Gold- 

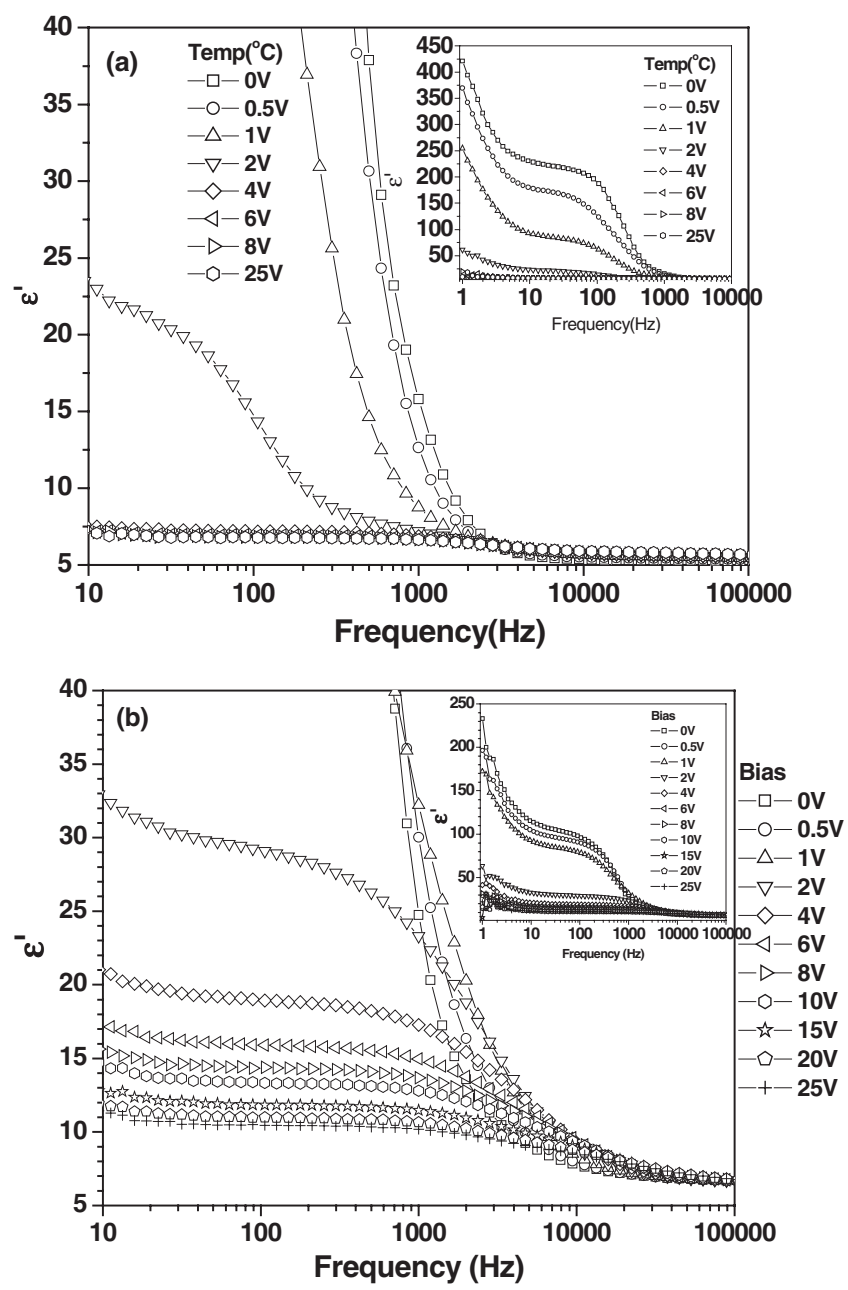

FIG. 3. Effects of bias voltage on the suppression of dielectric permittivity $\left(\varepsilon^{\prime}\right)$ at room temperature in (a) ethanol doped BDH 764E and (b) pure BDH 764E. The inset shows the overall behavior of $\varepsilon^{\prime}$ from 0 to $25 \mathrm{~V}$.

stone mode) in $\mathrm{SmC}^{*}$ phase appears up to $23{ }^{\circ} \mathrm{C}$ above the critical temperature for pure FLC material as shown in Fig. 2(a). This means that the transition temperature of ferroelectric $\mathrm{SmC}^{*}$ to paraelectric SmA phase is shifted by $23{ }^{\circ} \mathrm{C}$ by doping ethanol in the ELC material. Even the relaxation frequency of Goldstone mode is shifted to $51{ }^{\circ} \mathrm{C}$ temperature as seen in Fig. 2(b).

Above $51{ }^{\circ} \mathrm{C}$ temperature, the permittivity due to soft mode appears which is strongly temperature dependent as in case of conventional SmA phase. ${ }^{20}$ It should be mentioned here that the texture observation also suggests that the dechiralization lines disappear at $51{ }^{\circ} \mathrm{C}$ temperature, suggesting that the transition temperature has shifted from 28 to $51{ }^{\circ} \mathrm{C}$ temperature. This shift in the transition temperature from ferroelectric $\left(\mathrm{SmC}^{*}\right)$ to paraelectric $(\mathrm{SmA})$ phase can be varied depending upon the concentration of the ethanol dopant in the ELC material, but in the present study the highest temperature shift achieved was $23^{\circ} \mathrm{C}$.

The bias dependent behavior of $\varepsilon^{\prime}$ in ethanol added ELC material at lower frequencies is shown in Fig. 3(a). As seen in the figure the permittivity due to Goldstone mode gets completely suppressed at $4 \mathrm{~V}$ bias and no further change appears in the permittivity $\left(\varepsilon^{\prime}\right)$, whereas the permittivity $\left(\varepsilon^{\prime}\right)$ in pure BDH 764E ELC material does not get suppressed even at high bias field of $25 \mathrm{~V}$ as shown in Fig. 3(b). If one sees carefully at lower frequency range the static permittivity $\left(\varepsilon^{\prime}\right)$ behavior in pure and ethanol doped BDH 764E materials, the static permittivity in ethanol doped material is around 5 and in pure ELC material it is 12 . This means that one can still suppress the permittivity in pure ELC material by applying a higher bias field. This clearly shows that ethanol molecules are responsible for arranging the liquid crystal molecules uniformly in the layer structure which results in the suppression of dielectric permittivity $\left(\varepsilon^{\prime}\right)$ due to Goldstone mode (phason mode) at very low field, whereas in pure ELC material the static permittivity could not be suppressed even at a bias field of $25 \mathrm{~V}$ due to the randomization of the molecules in the layers. ${ }^{18}$ Analysis of Cole-Cole plots of the ethanol doped BDH 764E [Figs. 4(a)-4(c)] at 25, 49, and $53{ }^{\circ} \mathrm{C}$ temperatures reveals that single collective processes are observed in deep $\mathrm{SmC}^{*}$ and $\mathrm{SmA}$ phases. The low frequency values in Fig. 4 (right hand side) are due to the conductivity effect, according to the last term of Eq. (4). However, two processes, Goldstone mode and soft mode, are observed at $49{ }^{\circ} \mathrm{C}$ near ferroelectric to paraelectric phase transition without suppressing the Goldstone mode unlike in case of conventional FLCs. This means that after doping ethanol, these processes are visible without any bias application, which indicates the reduction in randomization of molecules.

Figure 5 shows the variation in ratio of polarization and tilt angle with respect to temperature. The relation between $P / \theta$ and pitch value of the material is given as follows: ${ }^{21}$

$$
P / \theta=\chi(f q+z),
$$

where $q=2 \pi / L$ ( $L$ is pitch), $\chi$ is dielectric susceptibility, and $f$ and $z$ are the flexoelectric and piezoelectric coupling coefficients between $P$ and $\theta$. In case of pure ELC, the fall of $P / \theta$ is faster than for ethanol doped ELC. The behavior of helicoidal pitch of the material may depend on the other parameters also, such as $f, \chi$, and $z$. The appearance of soft mode without any bias application in $\mathrm{SmC}^{*}$ phase near $T_{c}$ as explained above can be correlated with this gradual fall in the doped ELC. This gradual fall in doped ELC suggests that molecules are more aligned because of reduction in phason fluctuations.

Figure 6 shows the inverse of dielectric strength and relaxation behavior of soft mode at $10 \mathrm{~V}$ bias in ferroelectric $\left(\mathrm{SmC}^{*}\right)$ and paraelectric $(\mathrm{SmA})$ phases in ethanol doped ELC material. The behavior shows that the material obeys Curie-Weiss law, which was not obeyed in case of pure ELC as observed in our earlier studies. ${ }^{15,16}$ This means that the nature of material has been changed and ferroelectric to paraelectric phase transition is extended from 28 to $51{ }^{\circ} \mathrm{C}$ just by doping ethanol in the ELC material. At $10 \mathrm{~V}$ bias in $\mathrm{SmC}^{*}$ phase there is no absorption peak in the whole $\mathrm{SmC}^{*}$ phase, suggesting that the Goldstone mode is suppressed completely and soft mode appears near transition temperature of $\mathrm{SmC}^{*}$ and $\mathrm{SmA}$ phases, ${ }^{22,23}$ as shown in Fig. 4. It is worth mentioning here that in pure ELC material such behavior of soft mode near transition temperature was not observed in $\mathrm{SmC}^{*}$ and $\mathrm{SmA}$ phases due to the randomization of liquid crystal molecules. The increase in the transition tem- 

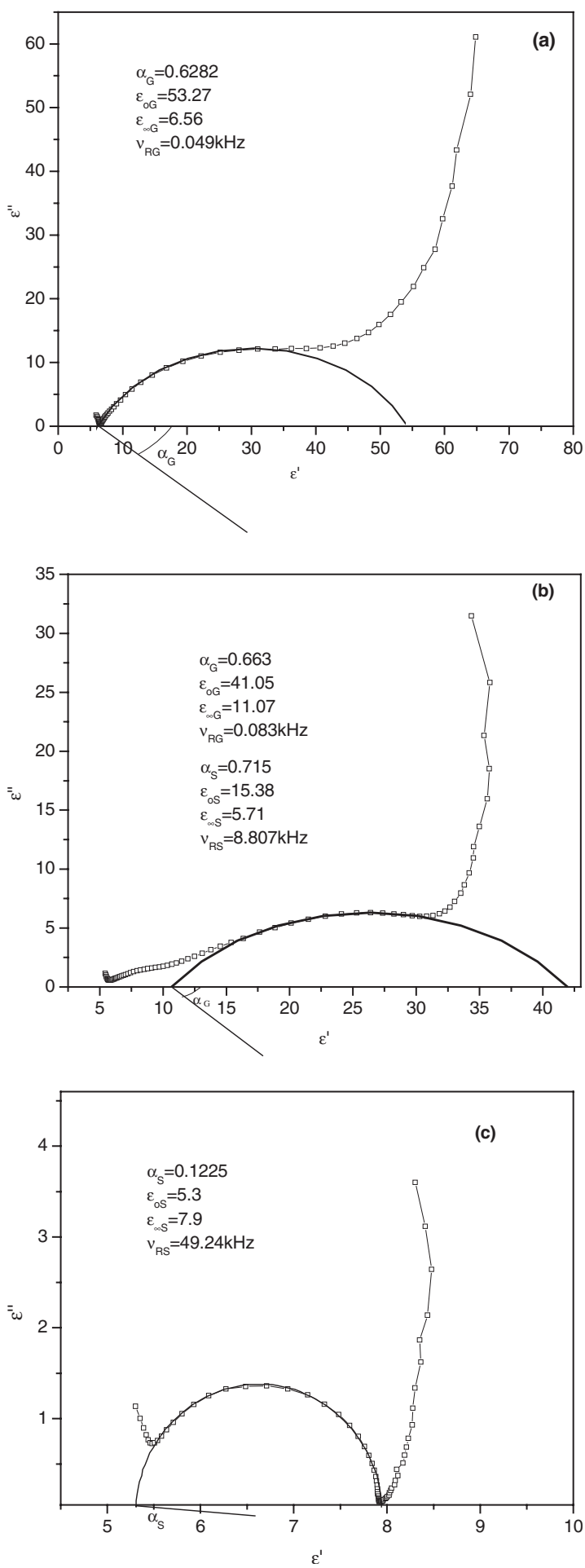

FIG. 4. Cole-Cole plots of ethanol doped BDH 764E at (a) 25, (b) 49, and (c) $53{ }^{\circ} \mathrm{C}$.

perature of $\mathrm{SmC}^{*}$ is due to the realignment of randomized liquid crystal molecules which contribute to the phase fluctuation mode in ferroelectric and paraelectric phase of liquid crystal material. This degradation in randomization of liquid crystal materials in $\mathrm{SmC}^{*}$ and $\mathrm{SmA}$ phases is due to doping of ethanol in the material. Since ethanol has dipole moment of $1.86 \mathrm{D}$, it can help to pursuit dipole-dipole interaction with liquid crystal materials. When this interaction comes into picture it dominates over the molecular interaction or

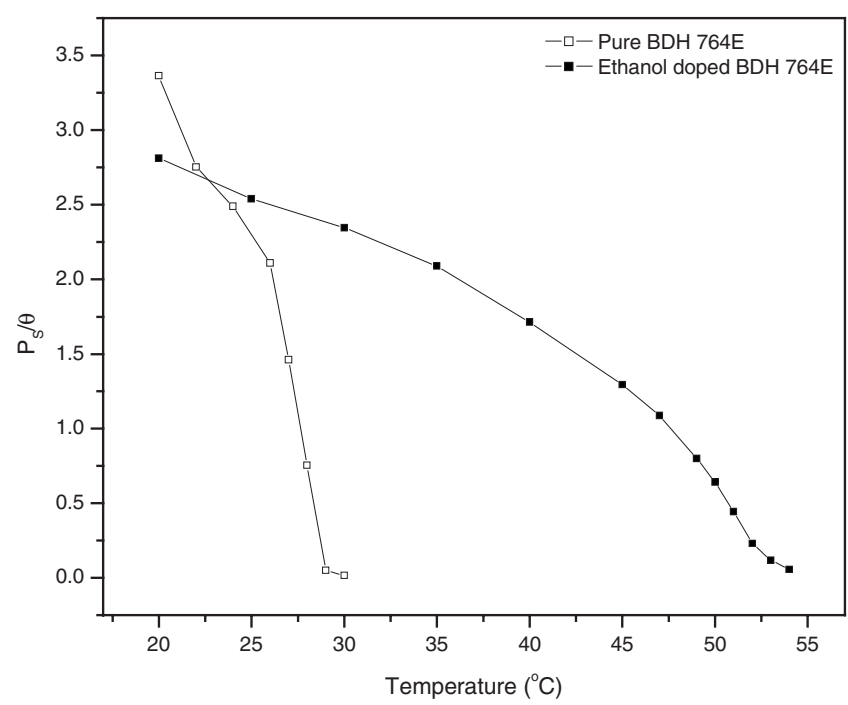

FIG. 5. Ratio of $P / \theta$ with respect to temperature $\left({ }^{\circ} \mathrm{C}\right)$ in pure $\mathrm{BDH} 764 \mathrm{E}$ (open square) and ethanol doped BDH 764E (closed square).

random fluctuations and hence aids in realignment of the randomizations. Once the FLC materials have less amount of randomization, then they behave as if they are in $\mathrm{SmC}^{*}$ phase and obey the Curie-Weiss law near the transition temperature of ferroelectric to paraelectric phase.

\section{CONCLUSIONS}

It can be concluded that the transition temperature of ferroelectric to paraelectric phase can be increased by doping ethanol in ELC materials. The randomization of the liquid crystal in de Vries ELC material can be adjusted by doping ethanol in the material and made uniform in the smectic layer structure, which would help to align ELC material easily. This work opens up new ways to implement FLCs for future applications at higher temperature and to understand the phase transition behavior in these materials. In addition

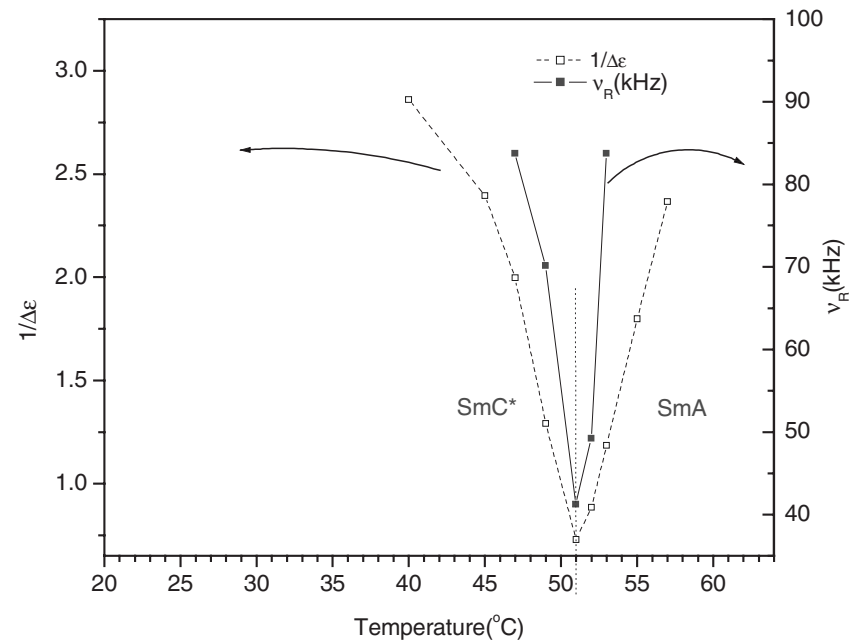

FIG. 6. Behavior of inverse of dielectric strength (open square) and relaxation frequency (closed square) at a bias of $10 \mathrm{~V}$ in $\mathrm{SmC}^{*}$ and $\mathrm{SmA}$ phases near $T_{c}$ which confirms the validity of Curie-Weiss law. 
to these implications, the study may also be useful for understanding the interaction between the ethanol and molecules of liquid crystal.

\section{ACKNOWLEDGMENTS}

The authors sincerely thank Dr. Vikram Kumar, Director, National Physical Laboratory, for continuous encouragement and interest in this work. The authors are thankful to S. S. Bawa, S. Kaur, and Ajay Kumar for fruitful discussions. They are thankful to the Department of Science and Technology (DST), New Delhi for supporting this work under Project No. SR/S2/CMP-03/2005. The authors A.M., A.C., and J.P. are thankful to Council of Scientific and Industrial Research (CSIR), New Delhi for financial assistance.

${ }^{1}$ S. Garoff and R. B. Meyer, Phys. Rev. A 19, 338 (1979).

${ }^{2}$ S. Garoff and R. B. Meyer, Phys. Rev. Lett. 38, 848 (1977).

${ }^{3}$ R. B. Meyer, L. Liebert, L. Strzelecki, and P. Keller, J. Phys. (Paris), Lett. 30, 69 (1975).

${ }^{4}$ G. Andersson, I. Dahl, W. Kuczynski, S. T. Lagerwall, K. Skarp, and B. Stebler, Ferroelectrics 84, 285 (1988).

${ }^{5}$ G. Andersson, I. Dahl, L. Kornitov, S. T. Lagerwall, K. Skarp, and B. Stebler, J. Appl. Phys. 66, 4983 (1989).

${ }^{6}$ P. A. William, N. A. Clark, M. B. Ros, D. M. Walba, and M. D. Wand, Ferroelectrics 121, 143 (1991).

${ }^{7}$ S. Diele, P. Brand, and H. Sackman, Mol. Cryst. Liq. Cryst. 16, 105
(1972).

${ }^{8}$ A. de Vries, Mol. Cryst. Liq. Cryst. 41, 27 (1977).

${ }^{9}$ A. de Vries, A. Ekachai, and N. Spielberg, Mol. Cryst. Liq. Cryst. Lett. 49, 143 (1979).

${ }^{10}$ B. R. Ratna, R. Shashidhar, G. G. Nair, S. K. Prasad, Ch. Bahr, and G. Heppke, Phys. Rev. A 37, 1824 (1988).

${ }^{11}$ M. D. Radcliffe, M. L. Brostrom, K. A. Epstein, A. G. Rappaport, B. N. Thomas, R. Shao, and N. A. Clark, Liq. Cryst. 26, 789 (1999).

${ }^{12}$ F. Giesselmann, P. Zugenmaier, I. Dierking, S. T. Lagerwall, B. Stebler, M. Kaspar, V. Hamploya, and M. Glogarova, Phys. Rev. E 60, 598 (1999).

${ }^{13}$ M. S. Spector, P. A. Heiney, J. Naciri, B. T. Weslowski, D. B. Holt, and R. Shashidhar, Phys. Rev. E 61, 1579 (2000).

${ }^{14}$ Y. P. Panarin, V. Panov, O. E. Kalinovskaya, and J. K. Vij, J. Mater. Chem. 9, 2967 (1999).

${ }^{15}$ S. Kaur, A. K. Thakur, A. Choudhary, S. S. Bawa, A. M. Biradar, and S. Annapoorni, Appl. Phys. Lett. 87, 102507 (2005).

${ }^{16}$ A. K. Thakur, A. Choudhary, S. Kaur, S. S. Bawa, and A. M. Biradar, J. Appl. Phys. 100, 034104 (2006).

${ }^{17}$ R. B. Meyer and R. A. Pelcovits, Phys. Rev. E 65, 061704 (2002).

${ }^{18}$ C. C. Huang, S. T. Wang, X. F. Han, A. Cady, R. Pindak, W. Caliebe, K. Ema, K. Takekoshi, and H. Yao, Phys. Rev. E 69, 041702 (2004).

${ }^{19}$ A. Mikulko, M. Marzec, S. Wrobel, J. Przedmojski, R. Douali, Ch. Legrand, R. Dabrowski, and W. Haase, Chem. Phys. Lett. 431, 289 (2006).

${ }^{20}$ A. K. Thakur, G. K. Chadha, S. Kaur, S. S. Bawa, and A. M. Biradar, J. Appl. Phys. 97, 113514 (2005).

${ }^{21}$ C. C. Huang and S. Dumrongrattana, Phys. Rev. A 34, 5020 (1986).

${ }^{22}$ J. Pavel and M. Glogarova, Ferroelectrics 121, 45 (1991).

${ }^{23}$ F. Gouda, G. Andersson, S. T. Lagerwall, K. Skarp, B. Stebler, T. Carlsson, B. Zeks, C. Filipic, and A. Levstik, Liq. Cryst. 6, 219 (1989). 